\title{
IES
}

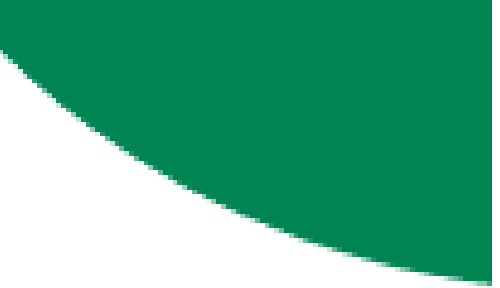

\section{WAITING LISTS, WAITING TIMES AND ADMISSIONS: AN EMPIRICAL ANALYSIS AT Hospital AND General Practice LeVEl}

Frank Windmeijer Hugh Gravelle Pierre Hoonhout 


\section{Waiting Lists, Waiting Times and Admissions: an Empirical Analysis at Hospital and General Practice}

\section{Level}

Frank Windmeijer $^{\mathrm{a}^{*}}$, Hugh Gravelle $^{\mathrm{b}}$, Pierre Hoonhout ${ }^{\mathrm{c}}$

${ }^{a}$ Centre for Microdata Methods and Practice, Institute for Fiscal Studies, UK

${ }^{\mathrm{b}}$ National Primary Care Research and Development Centre, Centre for Health

Economics, University of York, UK

${ }^{\mathrm{c}}$ University College London, $U K$

\section{Summary}

We report an empirical analysis of the responses of the supply and demand for secondary care to waiting list size and waiting times. Whereas previous empirical analyses have used data aggregated to area level, our analysis is novel in that it focuses on the supply responses of a single hospital and the demand responses of the GP practices it serves, and distinguishes between outpatient visits, inpatient admissions, daycase treatment and emergency admissions. The results are plausible and in line with the theoretical model. For example: the demand from practices for outpatient visits is negatively affected by waiting times and distance to the hospital. Increases in waiting times and waiting lists lead to increases in supply; the supply of elective inpatient admissions is affected negatively by current emergency admissions and positively by lagged waiting list and waiting time. We use the empirical results to investigate the dynamic responses to one off policy measures to reduce waiting times and lists by increasing supply.

JEL Classification: I11, H42

Keywords: waiting time; waiting list; hospital admissions

\footnotetext{
Correspondence to Institute for Fiscal Studies, 7 Ridgmount Street, London WCIE 7AE, UK. Email:
} f.windmeijer@ifs.org.uk 


\section{Introduction}

Although elective care in the National Health Service has been rationed by waiting time since the foundation of the NHS in 1948 there has been little econometric analysis of the way in which supply and demand respond to waiting times and waiting lists. This paper describes the results of an empirical analysis of the responses of inpatient, outpatient and emergency admissions to waiting list size and waiting times using data from a single hospital and the practices it serves.

The first part of the analysis focuses on the hospital level supply of outpatient visits, inpatient admissions, daycase admissions and emergency admissions. Since the theoretical models suggest that past behaviour and conditions influence future responses and outcomes, we utilize time series data at the hospital level to estimate supply responses to measures of current and past waiting times, list sizes and activity levels.

The second part examines the demand side by modelling the outpatient referral rates of the GP practices served by the hospital and their response to outpatient waiting times. As the data are repeated time series observations at GP practice level, we have a panel dataset allowing us to estimate dynamic models controlling for unobserved, fixed, GP heterogeneity in referral rates.

We then bring results from the two analyses together to perform simulations of the impact of temporary increases in activity levels resulting from one off policy initiatives. We increase the number of elective inpatient and day-case admissions exogenously by $10 \%$, sustained for three months, and calculate the responses to the various waiting measures that subsequently feed back into activity levels. The paths of the waiting measures and activity levels is charted for 21 months after the exogenous increases in activity levels on the assumption that all responses remain as they were estimated from past behaviour. These give insights of how the system responds to various pressures, and how long it takes for it to be back at its original path. For example, for the aggregate supply and demand at the particular hospital we 
find that the system is back to its original levels by 21 months after the initial three months sustained $10 \%$ increases.

There is a limited empirical literature on demand and supply response to waiting lists and waiting time. Martin and Smith (1999) estimated a supply and demand model using aggregate data on hospital utilization at a small area (ward) level in England for the years 1991 and 1992. The demand and supply equations are identified by exclusion restrictions and the models estimated by instrumental variables techniques. Gravelle, Smith and Xavier (2003) estimate demand and supply models using aggregate hospital utilization data at the more aggregated English Health Authority level for 24 quarters during the years 1987 to 1993. Whereas Martin and Smith (1999) only had one measure of hospital utilization, Gravelle, Smith and Xavier (2003) use separate additions and admissions measures for demand and supply respectively. Our study builds on this earlier econometric work in two respects. First, we model the admissions process in more detail by distinguishing amongst outpatient visits, inpatient admissions, day cases and emergency admissions. Second our data permit us to use the appropriate decision units when modeling demand and supply, namely general practices and the hospital. ${ }^{\mathrm{i}}$

A related, non-econometric, literature, suggests that NHS waiting lists are subject to the power laws of complexity theory. Smethurst and Williams (2001) and Papadopoulos et al. (2001) argue that the NHS is self-regulating, and governed by complex feedback mechanisms. Our economic approach allows us to unravel these "black-box" relationships by modeling the feedbacks explicitly. By using the estimated system dynamics we can calculate responses to shocks to the system, as in Van Ackere and Smith (1999) and Smith and Van Ackere (2002).

Section 2 sketches theoretical models of demand, supply, waiting time and waiting lists, which underpin the specification of the empirical models, and describes the data and methods. Section 3 presents the time series analysis of the models for supply, waiting times and waiting lists for the aggregate series of the hospital. Section 4 reports on the panel data analysis of the model for demand, the realised GP outpatient 
visits rates at the hospital. These estimation results are combined in a simulation exercise as described above in Section 5. Section 6 concludes.

\section{Modelling the market}

The setting for the paper is the Ayrshire and Arran Health Board (AAHB) in Scotland which covers a population of approximately 375,000 residents. The population is slightly older and more deprived than the Scottish average and has some of the highest unemployment rates in the UK (Arbuthnot, 1999). The population is spread across rural and urban areas, as well as two island communities. It is served by 61 general practices and the Acute Trust largely provides care from two main District General Hospital sites, one of which is the focus of our study.

\subsection{Demand and supply}

The specification of the empirical models is guided by the line of theoretical models of the NHS which derive from Lindsay and Feigenbaum (1984), especially Martin and Smith (1999), Gravelle, Sutton and Dusheiko (2002) and Gravelle, Smith and Xavier (2003). (See Cullis, Jones and Propper (2000) for a survey of the literature). The NHS has a list system with gatekeeping general practitioners: patients must join the list of a GP and the only access to NHS elective care is via a referral by their GP.

Patients do not pay for NHS hospital care but they do have to wait significant lengths of time for elective care. If a GP decides that a patient could benefit from elective care she refers the patient to see a specialist hospital consultant at a hospital outpatient clinic. The patient will join the waiting list for outpatient appointments and will be seen by the consultant after a delay of some weeks (the median wait for outpatient appointments in our data is 31 days). If the consultant decides that the patient should receive elective hospital care, the patient is then placed on the waiting list for elective admissions (either for day case treatment or for inpatient treatment) and will be treated after a further delay (the median time on the inpatient elective admissions waiting list in our data was 66 days). Since patients must incur initial financial and time costs in attending the outpatient clinic, increases in either the waiting time for an 
outpatient appointment or the waiting time for admissions after being seen by the consultant will reduce the demand for elective care. Thus the demand function $\left(D_{O V t}^{j}\right)$ for outpatient visits by practice $j$ at time $t$ and the total number added to the outpatient waiting list $\left(D_{O V t}\right)$ are

$$
D_{O V t}^{j}=D_{O V}\left(w_{O V t}^{g}, w_{I A t}^{g}, w_{D A t}^{g}, z_{t}^{j}\right), \quad D_{O V t}=\sum_{j} D_{O V t}^{j}
$$

where $w_{O V t}^{g}, w_{I A t}^{g}$ and $w_{D A t}^{g}$ are the waiting times for outpatient appointments $(O V)$, elective inpatient admissions (IA) and daycase elective admissions (DA) perceived by general practitioners and patients at the date the patient consults the general practitioner. $z_{t}^{j}$ is a vector of demand shifters which we discuss in more detail in Section 4 where we estimate practice level demand functions.

The probability that a patient seen in the outpatient clinic is placed on the inpatient or daycase waiting list by the consultant depends on the waiting times for these types of elective care and the patient's capacity to benefit from elective care. Hence the numbers added to the elective waiting lists in period $t$ are

$$
D_{I A t}=D_{I A}\left(O V_{t}, w_{I A t}^{h}, w_{D A t}^{h}\right), \quad D_{D A t}=D_{D A}\left(O V_{t}, w_{I A t}^{h}, w_{D A t}^{h}\right)
$$

where the waiting times for day case and inpatient elective care $\left(w_{k t}^{h}, k=I A, D A\right)$ are those forecast by the hospital consultant and the patient when the patient is seen in the outpatient department at period $t$.

The hospital supplies four types of care: outpatient visits $\left(O V_{t}\right)$, elective inpatient admissions $\left(I A_{t}\right)$, elective day case admissions $\left(D A_{t}\right)$, and emergency inpatient admissions $\left(E M I A_{t}\right)$. The waiting lists for outpatient visits, inpatient elective admissions and daycase elective admissions evolve as

$$
W L k_{t}=W L k_{t-1}+D_{k t}-k_{t}-\delta_{k t}, \quad k=O V, I A, D A
$$

where the $\delta_{k t}$ are the numbers of patients who leave these lists in the period because they die or move away or decide to get care in the private sector.

We assume that the hospital decisions on emergency admissions and on the first three types of care are taken by two different decision makers with different preferences. 
The utility function the emergency admissions decision maker is

$$
u_{1}^{t}=u_{1}\left(E M I A_{t}, O V_{t}, I A_{t}, D A_{t}, w_{t}^{p}, W L_{t}^{p} ; w_{t-1}^{p}, W L_{t-1}^{p}\right)
$$

and for the elective care decision maker is

$$
u_{2}^{t}=u_{2}\left(O V_{t}, I A_{t}, D A_{t}, w_{t}^{p}, W L_{t}^{p} ; E M I A_{t}, w_{t-1}^{p}, W L_{t-1}^{p}\right)
$$

The hospital is an independent not for profit public sector trust which is obliged to break even taking one year with the next. It has a block contract with the AAHB to treat AAHB patients under which its revenue does not vary with the number of cases treated within a range specified in the contract. The numbers of cases treated enter the utility functions because of their effects on hospital costs or because decision makers care about the well-being of patients.

$w_{t}^{p}=\left(w_{O V t}^{p}, w_{I A t}^{p}, w_{D A t}^{p}\right)$ is a vector of the waiting time performance indicators for outpatient visits, elective inpatient and elective daycase admissions used by the NHS to reward the hospital. $W L_{t}^{p}$ is an analogous vector of waiting list performance indicators. The specification of preferences allows for the possibility that rewards depend on the change in the performance indicator or a weighted average of current and past indicators. The hospital decision makers care about the hospital's performance indicators because the NHS rewards good performance in ways which affect all decision makers, although the marginal utilities of particular managers from a given indicator may differ. For example, hospitals that perform well may be rewarded with preferential access to funds for capital investment or managers may have their salaries linked to the performance indicators. More drastically, managers whose hospitals fail to achieve performance targets may be fired.

We assume that the two hospital decision makers move sequentially with decisions on outpatient visits $O V_{t}$, elective inpatient $I A_{t}$ and elective day case admissions $D A_{t}$ being taken after decisions on emergency admissions. This captures the plausible notion that emergencies have the first call on the hospital's resources. As a consequence the elective decision maker's choices of $O V_{t}, I A_{t}$, and $D A_{t}$ are conditional on $E M I A_{t}$. 
Although there is an exogenous random demand for emergency care, the emergency admissions decision maker has some control over emergency admissions. For example, the thresholds for admitting certain types of emergency patients for observation can be varied as can lengths of stay. The emergency decision maker may or may not care about elective admissions and outpatient visits and may or may not take account of his choice of $E M I A_{t}$ on the elective decision maker's choice of outpatient visits and elective admissions.

\subsection{Data}

Table 1 provides summary statistics for the variables used in the empirical analysis of the supply and waiting list/times models. The monthly number of elective inpatient admissions (IA), elective day-case admission (DA) and emergency inpatient admissions (EMIA) are taken from the Scottish Health Data general acute inpatient/day-case record SMR01 dataset, which collects patient based data on inpatient and day-case episodes in general and acute wards. The length of stay for each episode is also recorded in this dataset, and the average length of stay (LOSM) is calculated as the average length of stay of all elective and emergency inpatient episodes during a calendar month.

The number of monthly first outpatient visits $(O V)$ is from the outpatient record dataset SMR00. This dataset collects patient based data on first attendance at outpatient clinics in all specialties (except A\&E).

The number of patients waiting for elective inpatient treatment (WLIA) or day-case treatment $(W L D A)$ are from census data that record the number of patients waiting at a given point in time at regular intervals, in general the end of a quarter. The census also has information on the length of time patients on the list at the time of the census have been waiting. From this we have calculated the mean waiting time for those patients waiting for inpatient treatment (WTIM) and the median waiting time (WTI5), and the mean and median waiting times for those patients waiting for day-case treatment (WTDM and WTD5 respectively). These quarterly observations on waiting times have also been transformed to monthly observations by linear interpolation. 
We have further constructed a variety of different waiting times variables to allow for the fact that the theoretical models are not specific about the precise form in which waiting time and waiting lists enter patient demand functions and decision-makers' supply functions. The SMR01 records how long patients have been on the inpatient or day-case waiting list before being admitted. These are the realised waiting durations from the time of being put on the waiting list, in contrast to the census waiting times that refer to the stock of patient waiting at that point in time. We refer to the realised waits as durations in order to distinguish them from the census waiting times. As these durations are taken from the SMR01 record dataset, the averages (WDIM and WDDM for inpatient and day-case respectively) and medians (WDI5 and WDD5) are calculated from the monthly admissions. Combining the census waiting list information and the data on the number of admissions we have constructed the waiting times to clear the list for inpatient and day-case treatments as $W T T C I_{t}=W L I A_{t} / I A_{t}$ and $W T T C D_{t}=W L D A_{t} / D A_{t}$ respectively.

The outpatient record dataset SMR00 contains information on how long patients have been waiting from making their initial appointment to their first outpatient visit. We have constructed the realised mean and median outpatient waiting durations (WDOM and WDO5).

\subsection{Estimation of hospital model}

Referring back to the model sketched in section 2.1, we see that the decision makers' choices of activity levels in period $t$ will depend on the variables they perceive as exogenous at time $t$. The precise properties of these supply functions will depend on the utility functions and on whether the decision makers are myopic or allow for the effects of current decisions on future performance indicators. (See Gravelle, Smith and Xavier (2003) for a discussion and an example of some comparative statics in a similar but simpler model). We do not specify precise forms for the waiting time variables in the demand functions for outpatient visits and additions to the elective lists, or in the utility functions but let these be determined by the data. For example, general practitioners could forecast elective inpatient waiting time by using the realised mean or median waits of patients admitted in the previous period (WDIM or 
WDI5), or they could estimate waiting times as the time to clear the inpatient elective waiting list at last period's output rate: $w_{I A t}^{g}=W T T C I_{t-1}=W L I A_{t-1} / I A_{t-1}$. Or they may use weighted lagged sums of these measures. Further, the periods defined by the administrative databases may not correspond to those in the demand and utility functions. Thus in general the reduced form supply functions will depend on lagged values of waiting times, waiting lists, and past supply decisions. In the case of $O V_{t}$, $I A_{t}, D A_{t}$ we also allow for the possibility that current emergency admissions $E M I A_{t}$ enter the supply functions.

In addition to the supply functions we also estimate equations for evolution of the waiting lists and for some of the waiting time measures. Although the equation for the list size (3) is an accounting identity we do not have data on the additions to the list $\left(D_{k t}\right)$ or those dropping off the list $\left(\delta_{k t}\right)$. Thus we estimate models of the form

$$
W L I A_{t}=f\left(W L I A_{t-1}, I A_{t}, O V_{t-j}\right) .
$$

for the inpatient waiting list and similarly for the day case waiting list. Data on the outpatient waiting list is not available.

In all models, we allow for flexibility of the lag length with which explanatory variables enter the equation. We allow for all activity rates, waiting list/times, length of stay and bed availability variables to enter all models.

Since patients can choose between the two hospitals in the Health Board the demand for outpatient visits at the hospital we study could depend on the waiting times and waiting lists at the other hospital. Hence decisions at the two hospitals could be interdependent in that a change in supply in one hospital will change its waiting times thereby changing demand at the other hospital and leading it to alter its supply decisions. We assume that the decision makers in the hospital we study take the actions of decision makers in the other hospital as exogenous. Thus we allow for the possible effects of admissions, waiting lists and waiting times in the other hospital on the study hospital by including them as exogenous variables in the models for supply, waiting lists and waiting times. None of the other hospital variables were significant 
in any of the models for the study hospital.

The models were initially estimated by OLS. We found that transformation into natural logarithms of all variables resulted in the best model specification with respect to standard specification tests like the RESET test. Thus all estimated coefficients can be interpreted as elasticities. The variables to be included in the model were selected using a forward selection procedure. A full set of year and month indicators were originally included in the model to guard against spurious time series correlations of the various variables and to allow for exogenous shifts in preferences. The number of year and month indicators were then reduced in a final step by removing insignificant year/month effects in order to increase the number of degrees of freedom in the models. As the errors of the various models are likely to be contemporaneously correlated, we estimate the resulting equations jointly using the Seemingly Unrelated Regression Estimator (SURE) in a final step.

\section{Results for hospital supply}

Tables $2 \mathrm{a}$ and $2 \mathrm{~b}$ present the final model specifications and the values of the estimated coefficients from the OLS and SURE estimation procedures respectively. The two estimation techniques result in very similar estimated coefficients and in the following we will discuss the specific values using the SURE results.

Elective inpatient admissions (IA) vary negatively with day-case admissions in the previous month (elasticity -0.45) reflecting the substitution of day cases for inpatient elective admissions. We interpret this as the substitution of day cases for elective admissions. An increase in the number of emergency admissions in the current month decreases the number of elective inpatient admissions (elasticity -0.55), which supports our suggestion in section 2.1 that emergency admissions take priority over electives. The positive elasticity with respect to outpatient visits 3 months ago $(0.21)$ shows that increases in demand lead directly to increases in supply. Supply also increases in response to increases in the lagged waiting time (elasticity 0.40) and the lagged waiting list (elasticity 0.46). The other variables in the $I A$ model are year and 
month effects, plus an indicator for an outlying observation in January 2000 (possibly due to policies introduced to counteract fears of computer system failure at the start of the millennium). The model estimated by OLS shows a high $\mathrm{R}^{2}$ and passes the RESET test for misspecification. The Durbin-Watson (DW) test further indicates that there is no serial correlation problem in the residuals.

The results for elective day-case admissions (DA) are qualitatively similar to those for elective inpatients. The negative elasticity $(-0.16)$ with respect to elective inpatient admissions again suggests that inpatient admissions and day cases are substituted for each other to relieve workload pressure. As with inpatient electives, increases in outpatient visits increase supply (elasticity 0.27). Both the inpatient median wait duration (elasticity 0.31 ) and the day-case mean wait (elasticity 0.13 ) increase supply. The main qualitative difference between elective inpatient and elective day cases is that emergency admissions have no direct effect on day-cases. This is plausible since an increase in emergency admissions will have more effect on the resources available for elective inpatient admissions than for day cases.

In the model for the number of emergency inpatient admissions (EMIA) an increased number of patients on the elective inpatient waiting list in the previous month decreases the number of emergency admissions with an elasticity of -0.09 . An increase in the realised elective inpatient median waiting durations in the previous month leads to an increase in the number of emergency admissions, the estimated elasticity being 0.04 . We interpret this as an indication that the hospital does have some control over its emergency admissions and it varies them to relieve pressure on elective admissions as shown by waiting list and waiting times for elective inpatients.

There is strong serial correlation in the model for outpatient visits $(O V)$, with an apparent three months cycle. An increased number of outpatient visits three month ago is associated with an increase in the current period, whereas increases in the last and one-before-last months are associated with a decrease in the number of outpatient visits in the current month. The lag pattern may be explained by workload smoothing. Supply also responds to outpatient median realised waiting durations (elasticity 0.14 ). 
The elective inpatient waiting list (WLIA) responds as expected to the one-month lagged waiting list (elasticity 0.78 ) and to the supply of elective inpatient admissions in the previous month (elasticity -0.17). The list increases when outpatient visits increase as some of these lead to patients being placed on the list, the elasticity being 0.09. Increases in day-case admissions, which are a substitute for inpatient elective admissions also reduce the list (elasticity -0.11 ). ${ }^{\text {ii }}$

In the model for the day-case waiting list (WLDA) the elasticity with respect to outpatient visits in the previous month is again positive (0.31) and somewhat larger than for the inpatient waiting list. The waiting list again exhibits serial correlation though with two lags of the list affecting the current list. Unexpectedly, the number of day-case admissions does not enter the empirical model for the day-case waiting list.

The four waiting time models are broadly similar. Three of the four waiting time measures (mean inpatient waiting time - WTIM; mean day-case waiting time WTDM; median outpatient waiting time duration - WDO5) exhibit positive serial correlation. Waiting times are also correlated with lagged waiting list measures, with the day case waiting time measure (WTDM) varying with the lagged inpatient waiting list as well as the day case waiting list, possibly reflecting the substitutability of daycases and inpatient electives. Increases in supply reduce WTIM and WTDM.

The limited number of other studies of the effects of waiting times on supply also find positive elasticities. Martin and Smith (1999) modeled supply responses for elective surgery using a 1991-2 cross section of 4985 wards with average populations of around 10,000. Using two stage least squares to allow for endogeneity of waiting times they found that supply (cases treated per head of ward population) was significantly positively related to the mean waiting time for patients from the ward (elasticity 2.93). In a more elaborate study which used a 6 year panel (1991/2-1997/8) of 5499 wards, and also disaggregated surgery into 7 broad specialities, Martin and Smith (2003) found that in 5 of the specialities supply was significantly positively related to current waiting time and in the other two the partial correlation was 
negative but insignificant. The elasticity of the supply of all specialities with respect to waiting time was 5.29. Gravelle, Smith and Xavier (2003) estimated a model for the supply of elective cases with a panel of 123 English Health Authorities over 24 quarters (1987Q2-1993Q1). Elasticities with respect to the 1 quarter lagged mean waiting timer were much smaller than those of Martin and Smith (1999, 2003) ranging from 0.08 to 0.18 . Our estimated elasticity of the supply of inpatient admissions with respect to the four month lag of mean waiting time is 0.40 and is therefore closer to those in Gravelle, Smith and Xavier (2003) than those of Martin and Smith (1999, 2003). Differences in estimation methods, units of analysis, setting, variable definitions and the timing of the studies make explanation of the differences in the estimates problematic but all have the same qualitative response of supply to waiting times.

\section{Outpatient Visits Rates - A Panel Data Analysis}

We next estimated models of demand by general practices for outpatient visits, some of which feed through into inpatient and day-case electives. The aim was to establish a demand response to waiting lists and/or waiting times at the hospital to complement the hospital supply models. We have available a panel of 61 GP practices with at most 19 quarterly observations, for the period 1997q2-2001q4. ${ }^{\text {iii }}$

The empirical model for the outpatient first visit rate by GP practice $j$ in period $t$ is

$$
O V R_{j t}=\alpha W D O M_{t-s}+\sum_{i} \beta_{i} x_{j t i}+\sum_{\ell=1} \gamma_{\ell} z_{j \ell}+\eta_{j}+v_{j t}
$$

$O V R$ is the outpatient visit rate per patient practice and WDOM is the mean realised waiting duration for first outpatient visits at the hospital. The vector $x_{j t}$ contain "need" variables that include the age/sex profile of the GP patient population, the fraction of deaths of patients aged 0-64 at the practice level, and the standardised illness ratio, which is the all age/sex standardised proportion with a limiting long-term illness using 1991 figures for the area where the GP practice is located. ${ }^{\text {iv }}$ The $z_{j}$ contain GP practice characteristics, including the distances to the study hospital and to the alternative hospital in the Health Board, whether the practice can dispense pharmaceuticals, 
whether he practice can perform minor surgery and the number of partners. We include year and quarter indicators in the model. Given the large number of practices it seems safe to assume that each practice takes hospital waiting times as unaffected by its decisions on referral so that it is unlikely that there is any simultaneous equation bias affecting the estimates of the effects of waiting times.

Table 3 presents a description of the variables used and their acronyms, together with some summary statistics. On average, just over $2 \%$ of GP patients have a first outpatient visit per quarter.

Table 4 presents estimation results for the GP-practice level data. These results are from a simple OLS regression (weighted by the number of patients per GP practice) on the pooled data. We find a negative response of the outpatient visits rate with respect to the mean waiting duration for outpatient visits at the hospital. A $10 \%$ increase in the mean waiting duration 2 quarters ago leads to a decrease of $3.2 \%$ in the GP level outpatient visits rate.

Inpatient and day-case waiting durations were also found to individually affect the $O V R$, as suggested by the theoretical model. However, none of the coefficients on the waiting variables were statistically significantly different from 0 when entered jointly, together with the mean outpatient visits waiting duration. The selection of the mean outpatient visits waiting duration provided the best fit to the data. We also included the waiting times at the other hospital in the Health Board but found no significant effects on demand for the study hospital.

There is no very clear pattern of demographic effects. With the fraction of the GP patient population aged 45-64 as reference point, the fractions aged 5-14 and 75-84 are associated with less outpatient visits. A higher fraction of females is associated with more outpatient visits. GP practices closer to the hospital and further away from the alternative hospital have higher outpatient visits rates. Dispensing GP practices have less referrals. Both the local need indicators, the standardised all-ages limiting long term sickness ratio in 1991, and the fraction of deaths in the GP patient 
population aged 0-64 are found to have an expected positive association with the outpatient visit rate.

As the mean outpatient waiting duration at the hospital is not correlated with the individual practice level characteristics, dropping these characteristics from the model does not alter the waiting duration elasticity much. Estimation of a model that excludes all variables except the outpatient waiting duration and time indicators results in an estimated elasticity of -0.30 with a standard error of 0.16 . When we include practice specific fixed effects, the waiting duration elasticity estimate is -0.09 with a standard error of 0.23 ; adding all the GP specific constants reduces the information available to estimate the waiting duration elasticity precisely.

It is interesting to assess the habit persistence of GPs. This could be modelled as practice fixed effects, i.e. some practices always have higher outpatient visits rates than others, or as a dynamic process, a high outpatient visit rate in the past leads to a higher outpatient visit rate in the present (see also Goddard and Tavakoli (1998)). A combination of these two processes is likely to be the appropriate model. Table 5 present OLS results of a dynamic model in the first column. The coefficient on the lagged outpatient visits rate is very high, 0.92, indicating that the GP outpatient visits rates are very persistent. This could be due to a mixture of habit persistence and unobserved GP practice characteristics. The results in the second column in Table 5 are the estimates from a dynamic panel data model estimated by IV-GMM, using the so-called "level" moment conditions (see Blundell and Bond (1998)) with two instruments for the lagged referral rate, viz. the first and second lag of the differenced referral rates. These instruments are valid under certain circumstances in dynamic models with unobserved GP heterogeneity. The coefficient on the lagged outpatient visits rate is now much lower, 0.32 , indicating that practice fixed behaviour dominates the persistence in the series. The (short-term) outpatient waiting duration elasticity is estimated as -0.32 in this IV model. The instrument validity is not rejected by the Sargan test, and the errors of the model in first differences display the proper MA(1) form. 
Although literature to date has examined the effect of waiting times on elective admissions and day-cases, rather than on the demand for outpatient visits, our estimated elasticities with respect to waiting time are not dissimilar. Papers which also examine practice level admission find elasticities of -0.25 for cataract surgery (Gravelle, Sutton and Dusheiko (2002) and -0.14 for all types of electives (Dusheiko et al, 2004). The somewhat higher elasticities in our models may reflect the possibility that the proportion of first outpatient visits which do not lead to elective care may be more responsive to waiting times than the more serious cases.

\section{Simulation of short term policy initiatives to reduce waiting}

In this section we present some simulations derived from the results of the hospital supply and the GP practice level demand results. Figure 1 charts the relationships and feedbacks between the various activity and waiting list/times variables found in the empirical analysis, including the GP outpatient visit rate response to outpatient waiting durations.

As all estimated coefficients are elasticities, we can set some exogenous changes in percentage levels, and calculate the results in terms of changed waiting lists/times and activity levels, also in percentages, for the months following the exogenous changes. Thus we can examine the effects of a short term policy aimed at reducing lists by say paying consultants to work extra sessions leading to an increase in inpatient admissions over a fixed period. We are interested in the changes in the future time paths of the endogenous variables (waiting times, waiting lists, supply) as the exogenous shock works its way through the system.

We simulate the effects of a 3 months sustained $10 \%$ increase in both inpatient elective admissions and day-case admissions. During this period the direct feedbacks between elective inpatients admissions, day-case admissions and outpatient visits are set to zero, but they are allowed to develop in the months after. The simulation is based on the SURE results. The GP outpatient visits rate elasticity with respect to outpatient waiting durations has been set equal to -0.4 , with a four-month lag, 
combining the estimation results in Tables 4 and 5. Figure 2 presents the monthly percentage changes in the various activity and waiting list/times series in the months after the initial 3 months of increased activity. The way to interpret these is that if for example the elective inpatient admissions are $-2 \%$, this means that activity levels are $2 \%$ below what they would otherwise have been.

The increased activity levels lead to a decrease in inpatient waiting list and inpatient, day-case and outpatient waiting times for the first 9-14 months. For the waiting durations, the largest relative effect is found for the mean day-case waiting time, which is almost $6 \%$ lower 3 months after the sustained increases in activity levels. The inpatient waiting list is about $6 \%$ lower than it would have been one month after the sustained increases. Because the model for day-case waiting lists does not contain the day-case activity levels, there is no decrease in the day-case waiting list. We will return to this issue below. Due to the decreased pressure on the system, there is a decrease in the number of inpatient and day-case admission for the first 8-11 months. Especially the number of elective inpatient admissions is almost $3.5 \%$ lower in months 6 and 7, whereas the number of day-case admissions decreases by around 1\% in the months 4-11. After this period, waiting lists and times start to become higher than they otherwise would have been, and inpatient and day-case activity have a positive response to this. There is a slight increase in the number of outpatient visits, peaking in month 12 at $1 \%$, and there is a slight initial increase in the number of emergency admissions. After about two years the system is almost back to its original levels.

In Figure 3, we impose an elasticity of the day-case waiting list size with respect to the number of day-case admissions equal to that of the inpatient elasticity, -0.17 . There now is of course a clear initial reduction in the day-case waiting list size, but this does not affect the results for the activity levels by much, as the day-case waiting list enters the model for activity levels only indirectly via the day-case waiting times. 


\section{Conclusions}

This paper has given a detailed account of time series analyses of empirical models for supply, waiting lists and waiting times at the hospital/specialty level for two hospitals in a Scottish region. A demand specification was also estimated using GP practice level information on the realised outpatient visits rate per GP practice over time. The results are generally consistent with the theoretical framework and the estimated elasticities have plausible values.

The estimation results were combined to perform a simulation exercise to assess the responses to activity levels and waiting measures to exogenous increases in the number of elective inpatient and day-case admissions. The simulation exercise takes account of the dynamic relationships and feedbacks found between the various activity levels and waiting measures. The specific results from our study will not of course carry over to other hospitals and other areas. Especially where there is a greater density of population and hence of hospitals we would expect that there will be interactions between providers to be taken account of. Nevertheless our analysis does show that it is possible to estimate economically sensible dynamic models of the supply and demand for hospital care. It also carries the general lesson that that oneoff policy interventions to reduce waiting times have complicated effects which are difficult to predict a priori without a formal model and which persist for a considerable time.

\section{Acknowledgements}

The paper is derived from a project commissioned by Ayrshire and Arran Health Board. Frank Windmeijer acknowledges further financial support from the ESRC, grant number H141251024, for the research forms part of the programme of research at the ESRC Centre for Economic Evaluation at IFS, a member of the ESRC Network of Evidence Based Policy and Practice. NPCRDC receives funding from the Department of Health. The views expressed are those of the authors and not necessarily of the funders. We thank John Forbes, Kirsten Major, Carol Propper, and Matthew Sutton for comments and suggestions, and Mike Muirhead from ISD 
Scotland for the supply of the data.

${ }^{\mathrm{i}}$ Windmeijer and Hoonhout (2004) also estimated supply models for some specialties.

ii The model for waiting lists as described in Section 2 is in the levels of the number of patients on the list and admitted and not in logs. Estimation of a model where the variables are not transformed into logs results in the same model specification.

${ }^{\text {iii }}$ Using monthly data would result in too many zero outpatient visits rates.

iv These variables were selected from various "need" indicators, including Arbuthnott indices, using standard model selection procedures. 


\section{References}

Ackere, van, A. and P.C. Smith (2002), A Note on the Integration of System Dynamics and Economic Models. Journal of Economic Dynamics and Control, 26, 1-10.

Arbuthnott, J. (1999), Fair Shares for All, Report of the National Review of resource Allocation for the NHS Scotland. http://www.scotland.gov.uk/library2/doc01/fsag-00.htm

Blundell, R. and S. Bond (1998), Initial Conditions and Moment restrictions in Dynamic Panel Data Models, Journal of Econometrics 87, 115-143.

Cullis, J. G., P.R. Jones, and C. Propper (2000), Waiting Lists and Medical Treatment, in A. J. Culyer and J. Newhouse (eds), Handbook of Health Economics. North Holland, Amsterdam.

Dusheiko, M., Gravelle, H., Jacobs, R., Smith, P.C. (2004), The effect of budgets on doctor behaviour: evidence from a natural experiment. May 2004. Department of Economics and Related Studies, Discussion Paper 2003/04. http://www.york.ac.uk/depts/econ/dp/2003.htm.

Goddard. J.A. and M. Tavakoli (1998), Referral Rates and Waiting Lists: Some Empirical Evidence, Health Economics 7, 545-549.

Gravelle, H., M. Dusheiko and M. Sutton (2002), Demand for Elective Surgery in a Public System: Time and Money Prices in the UK National Health Service, Journal of Health Economics 21, 423-449.

Gravelle, H., P.C. Smith, and A. Xavier (2003), Performance Signals in the Public Sector: The Case of Health Care, Oxford Economic Papers, 55, 81-103.

Lindsay, C.M. and B. Feigenbaum (1984), Rationing by Waiting Lists, American Economic Review 74, 404-417.

Martin, S. and P.C. Smith (1999), Rationing by Waiting Lists: an Empirical Investigation, Journal of Public Economics 71, 141-164.

Martin, S. and P.C. Smith (2003), Using Panel Data Methods to Model Waiting Times for National Health Service surgery, Journal Royal Statistical Society, Series A, 166, 369-397.

Papadopoulos M.C., M. Hadjitheodossiou, C. Chrysostomou, C. Hardwidge and B.A. Bell (2001), Is the National Health Service on the edge of chaos? Journal of the Royal Society of Medicine, 94, 613-616.

Smethurst D.P. and H.C. Williams (2001), Are Hospital Lists Self Regulating? Nature, 410, 652-653

Smith, P.C. and A. van Ackere (1999), Towards a Macro Model of National Health Service Waiting Lists, System Dynamics Review, 15, 225-252.

Windmeijer, F. and P. Hoonhout (2004), Capacity of the Health Care System: An Investigation of Activity and Constraints, Report for NHS Ayrshire and Arran Health Board. 


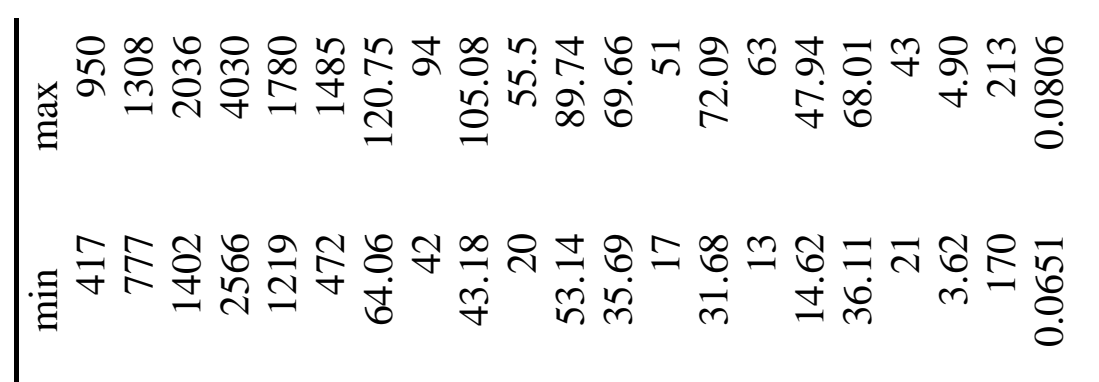

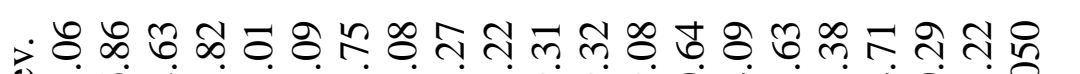

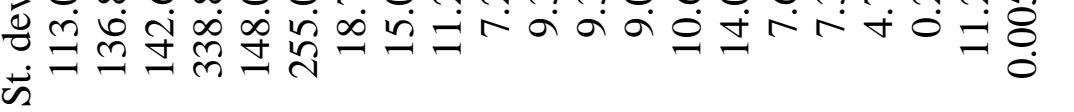

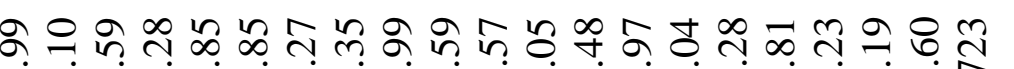

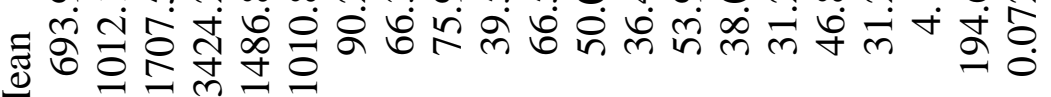
$\sum$

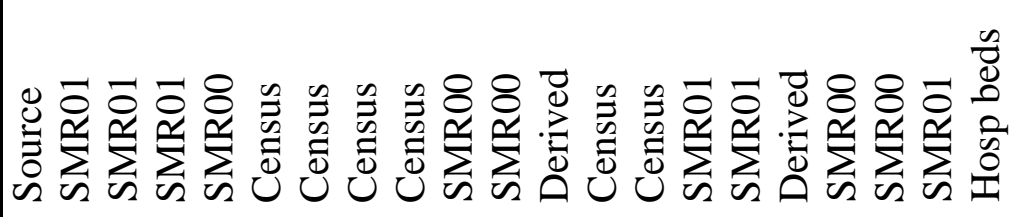

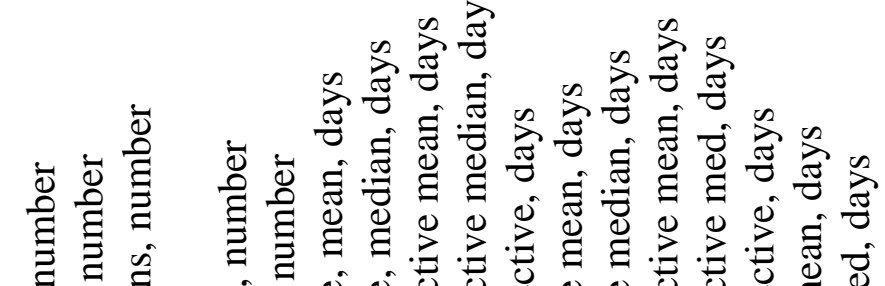

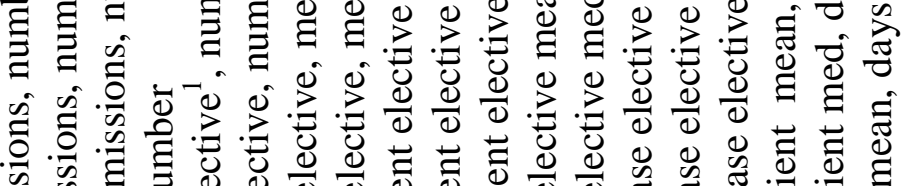
W.

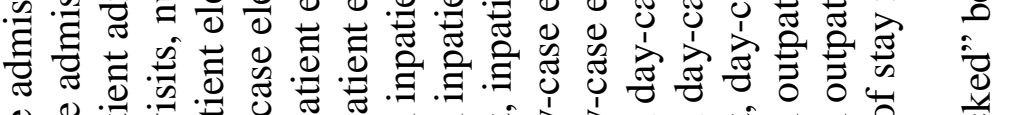

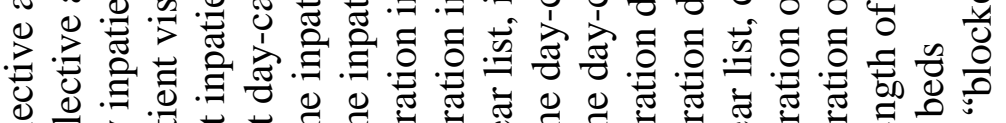

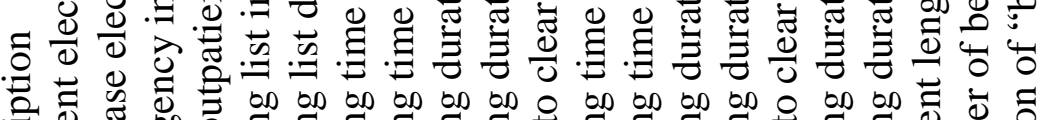

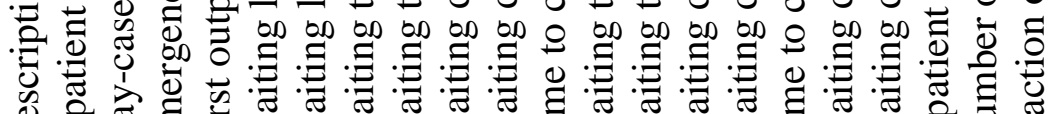

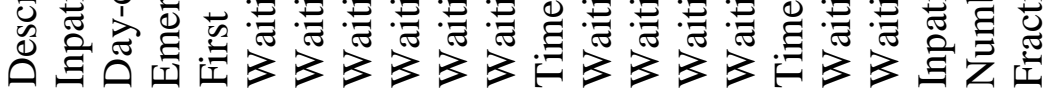




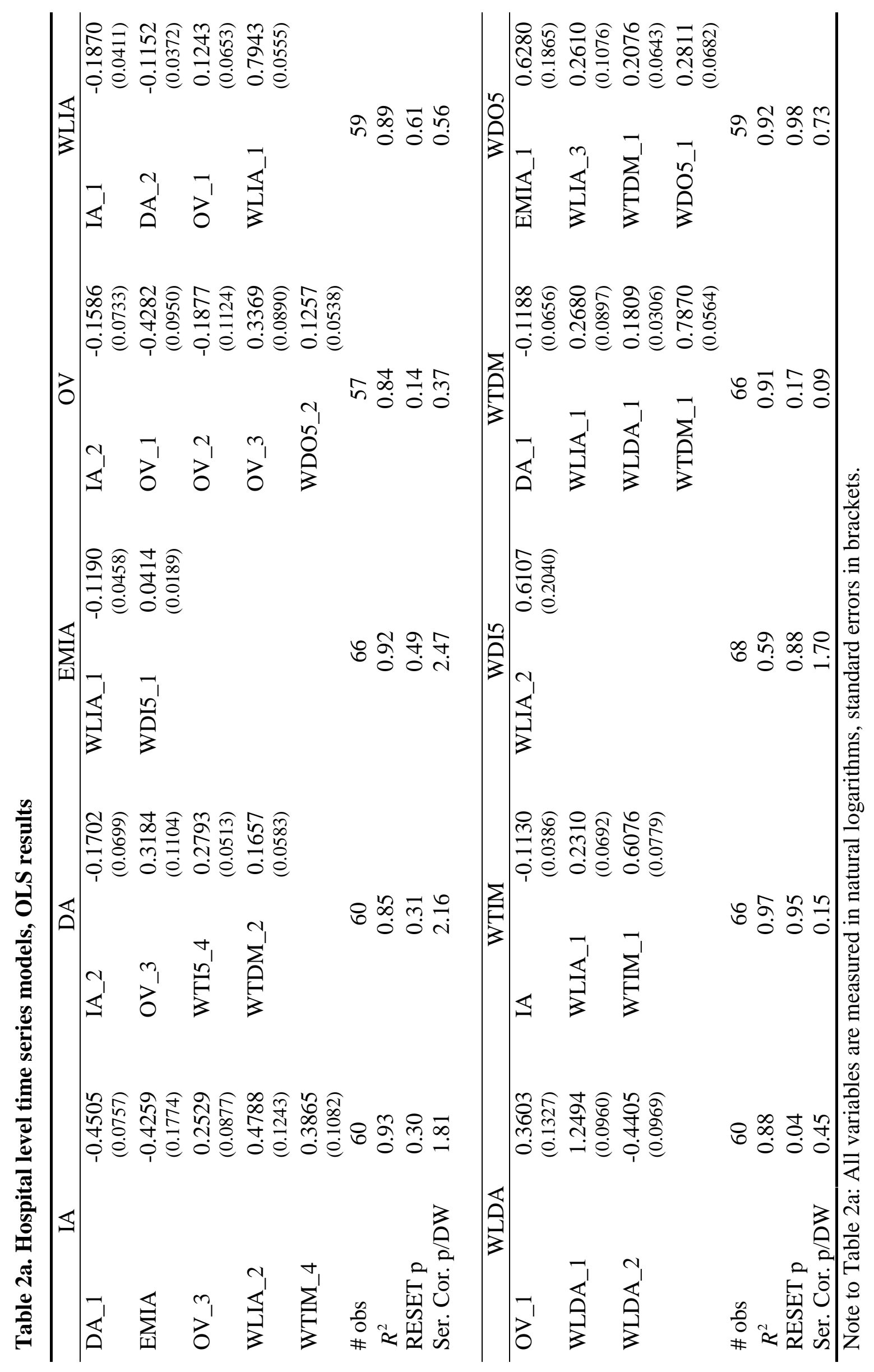




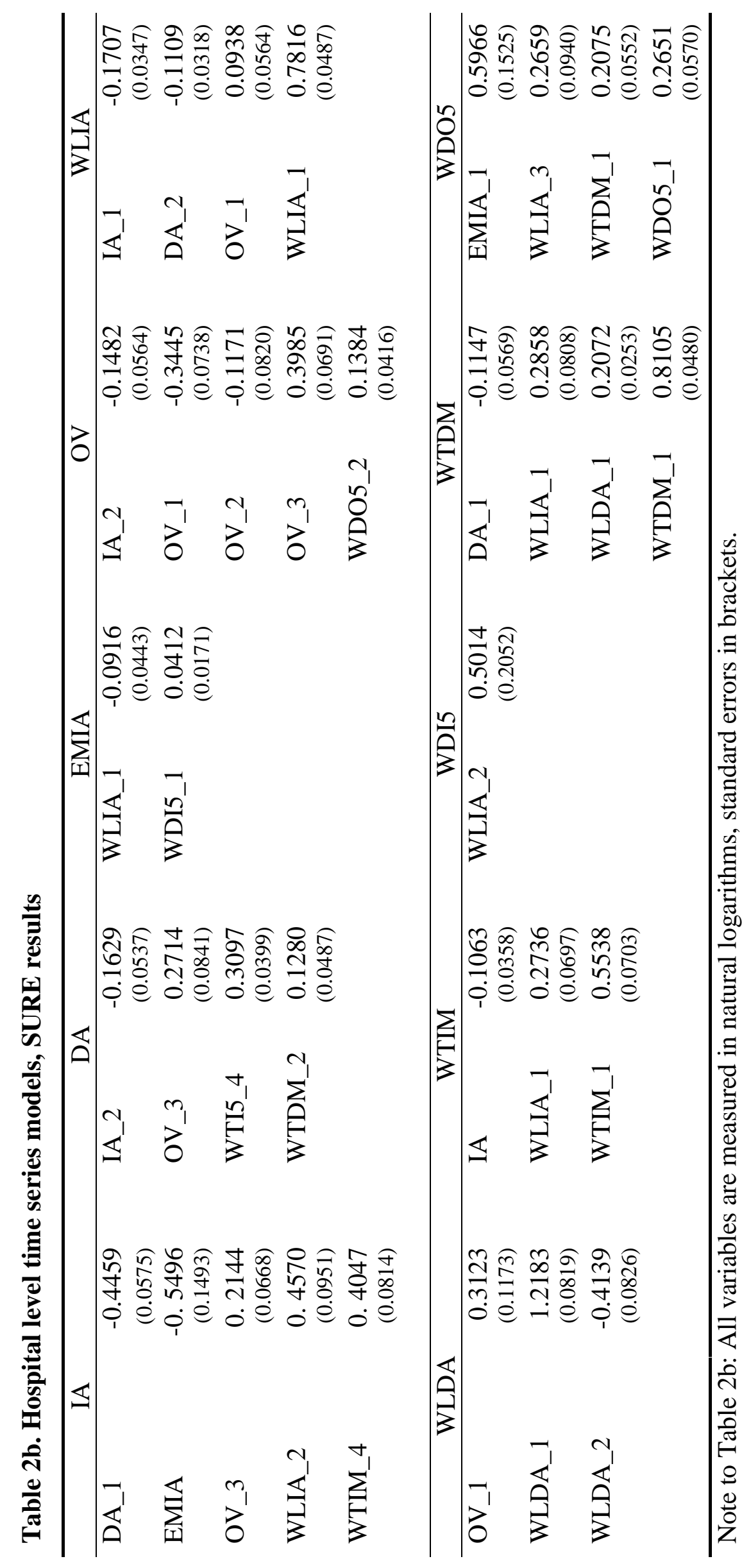


Table 3. Variable description and summary statistics of pooled data

\begin{tabular}{|c|c|c|c|}
\hline Variables & Description & Mean & St. Dev. \\
\hline OVR & Outpatient visits rate & .0228 & .0173 \\
\hline FRAGE01 & $\begin{array}{l}\text { Fraction of GP patients between } 0 \text { an } 1 \\
\text { years of age (x100) }\end{array}$ & 1.8974 & .4161 \\
\hline FRAGE24 & $2-4$ & 3.2802 & .6778 \\
\hline FRAGE514 & $5-14$ & 12.5393 & 1.8933 \\
\hline FRAGE1519 & $15-19$ & 6.2160 & .8439 \\
\hline FRAGE2024 & $20-24$ & 5.4411 & .8963 \\
\hline FRAGE2544 & $25-44$ & 28.6507 & 2.9269 \\
\hline FRAGE4564 & $45-64$ & 25.1598 & 2.4548 \\
\hline FRAGE6574 & $65-74$ & 9.3303 & 2.2763 \\
\hline FRAGE7584 & $75-84$ & 5.6095 & 1.6348 \\
\hline FRAGE85P & $85+$ & 1.8752 & .8285 \\
\hline FRFEMALE & Fraction of GP patients female & 51.0354 & 2.2568 \\
\hline DIST & $\begin{array}{l}\text { Distance from GP practice to hospital } \\
(\mathrm{km})\end{array}$ & 19.1182 & 12.5393 \\
\hline DIST_HOSP2 & $\begin{array}{l}\text { Distance from GP practice to } \\
\text { alternative hospital }(\mathrm{km})\end{array}$ & 20.8467 & 11.9634 \\
\hline D_DISPEN & Indicator for dispensing GP practice & .0504 & .2189 \\
\hline D_MINORS & $\begin{array}{l}\text { Indicator for GP practice performing } \\
\text { minor surgery }\end{array}$ & .7212 & .4485 \\
\hline PARTNERS & Number of partners & 4.0639 & 2.0897 \\
\hline SIR91 & Standardised Illness Ratio 1991 & 104.3237 & 25.0756 \\
\hline FRDEATHS_064 & Fraction of deaths, ages $0-64 * 100$ & .0682 & .0476 \\
\hline DFRDEATHS_064 & Zero deaths ages $0-64$ & .1154 & .3197 \\
\hline
\end{tabular}


Table 4. OLS results for GP level log outpatient visits rate

\begin{tabular}{|c|c|c|c|}
\hline Variables & Coeff & Rob Se & t-ratio \\
\hline \multicolumn{4}{|l|}{ Dep. Var. $L O V R$} \\
\hline LWDOM_2 & -.3168 & .1875 & -1.69 \\
\hline FRAGE01 & -.1414 & 1820 & 0.08 \\
\hline FRAGE24 & -.1416 & 1335 & -1.06 \\
\hline FRAGE514 & -.0250 & .0863 & -1.64 \\
\hline FRAGE1519 & 1305 & .1144 & -0.22 \\
\hline FRAGE2024 & -.0703 & 1340 & 0.97 \\
\hline FRAGE2544 & -.1414 & .0521 & -1.35 \\
\hline FRAGE4564 & - & & \\
\hline FRAGE6574 & .0005 & 1196 & 0.00 \\
\hline FRAGE7584 & -.3196 & .1055 & -3.03 \\
\hline FRAGE85P & -.0394 & .1903 & -0.21 \\
\hline FRFEMALE & .0575 & .0269 & 2.14 \\
\hline LDIST & -.6992 & .1136 & -6.15 \\
\hline LDIST_HOSP2 & .5738 & .0924 & 6.21 \\
\hline D_DISPEN & -.9107 & .3706 & -2.46 \\
\hline D_MINORS & .1948 & 1617 & 1.20 \\
\hline LPARTNERS & -.1882 & 1311 & -1.44 \\
\hline LSIR91 & .7290 & .3084 & 2.36 \\
\hline LFRDEATHS_064 & .0782 & .0340 & 2.30 \\
\hline DFRDEATHS_064=0 & .1631 & .1149 & 1.42 \\
\hline$Y 1997$ & -.1167 & .0675 & -1.73 \\
\hline$Y 1998$ & -.0325 & .0401 & -0.81 \\
\hline Y1999 & - & - & - \\
\hline$Y 2000$ & -.0223 & .0288 & -0.77 \\
\hline$Y 2001$ & .0674 & .0523 & 1.29 \\
\hline$Q 1$ & - & - & - \\
\hline$Q 2$ & -.0197 & .0133 & -1.48 \\
\hline$\widetilde{Q 3}$ & -.0182 & .0158 & -1.16 \\
\hline$Q 4$ & -.0133 & .0169 & -0.79 \\
\hline \#OBS & & 997 & \\
\hline \#GP practices & & 61 & \\
\hline Time period & & 1997q4-2001q4 & \\
\hline $\mathrm{R} 2$ & & 0.73 & \\
\hline RESET & & $161, \mathrm{p}$-value 0.00 & \\
\hline
\end{tabular}

Notes: The prefix $L$ indicates a logarithmic transformation of the variable.

Standard errors are robust to heteroskedasticity and general correlation over time of the residuals by GP practice 
Table 5. OLS and GMM results for GP level log outpatient visits rate, dynamic model

\begin{tabular}{|c|c|c|c|c|}
\hline \multirow[b]{2}{*}{$\begin{array}{l}\text { Variables } \\
\text { Dep. Var. } L O V R\end{array}$} & \multicolumn{2}{|c|}{ OLS } & \multicolumn{2}{|c|}{ IV Level } \\
\hline & Coeff & Rob Se & Coeff & Rob Se \\
\hline $\begin{array}{l}L O V R \_1 \\
L W D O M \_2\end{array}$ & $\begin{array}{r}.9247 \\
-.3693\end{array}$ & $\begin{array}{l}.0294 \\
.2043\end{array}$ & $\begin{array}{r}.3274 \\
-.3209\end{array}$ & $\begin{array}{l}.0570 \\
.1656\end{array}$ \\
\hline $\begin{array}{l}\text { \#OBS } \\
\text { \#GP practices } \\
\text { Time period } \\
\text { R2 } \\
\text { RESET }\end{array}$ & $\begin{array}{r}1997 q \\
0 \\
10.7\end{array}$ & 00 & $\begin{array}{r}93 \\
6 \\
1998 \mathrm{q} 1- \\
\text { Sargan } \\
\text { p-Ar1, 0.00, }\end{array}$ & $\begin{array}{l}4 \\
0.69\end{array}$ \\
\hline \multicolumn{5}{|c|}{$\begin{array}{l}\text { Notes: Other variables included as in Table } 4 . \\
\text { IV estimation results obtained with the Stata routine XTABOND2. }\end{array}$} \\
\hline
\end{tabular}




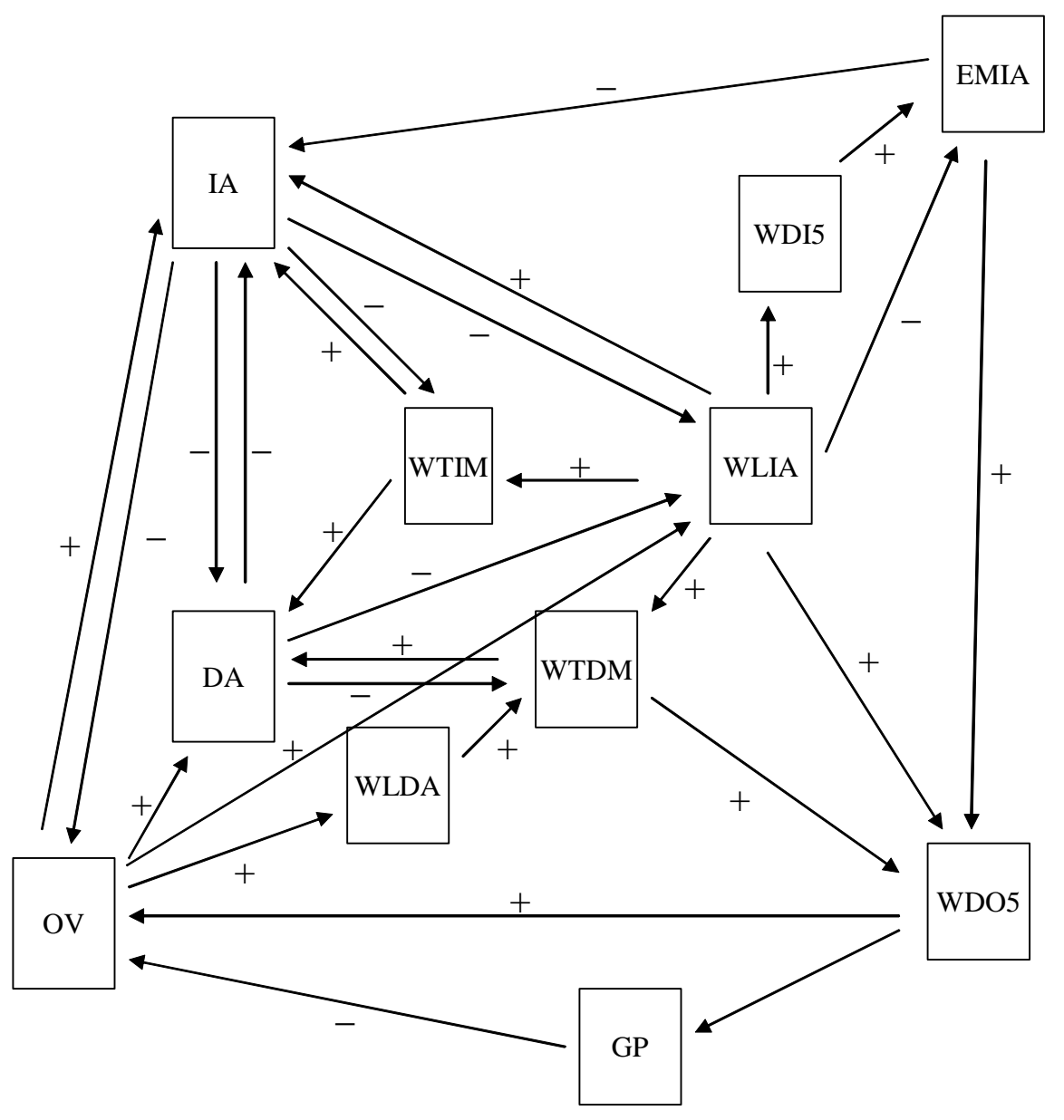

Figure 1. Flowchart of relationships in the empirical models. 

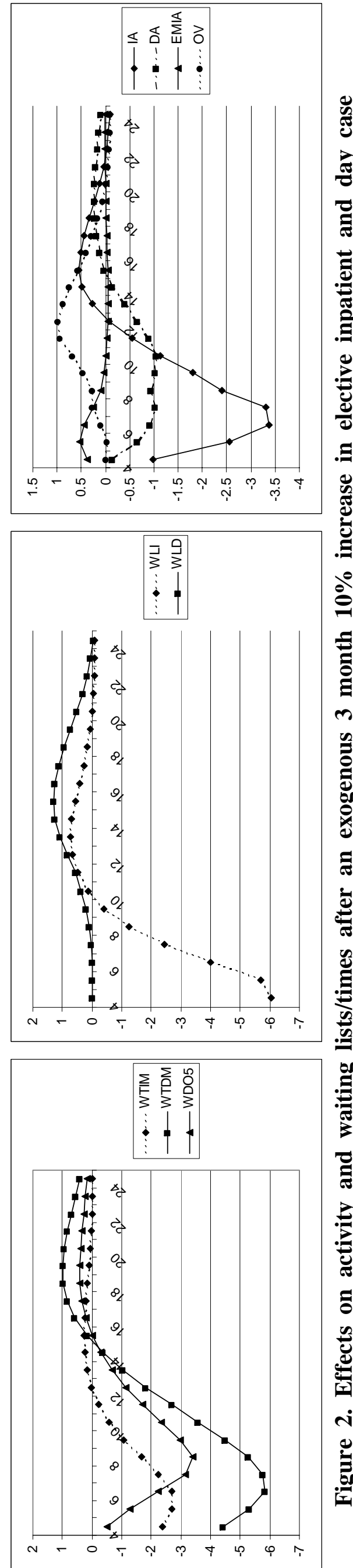

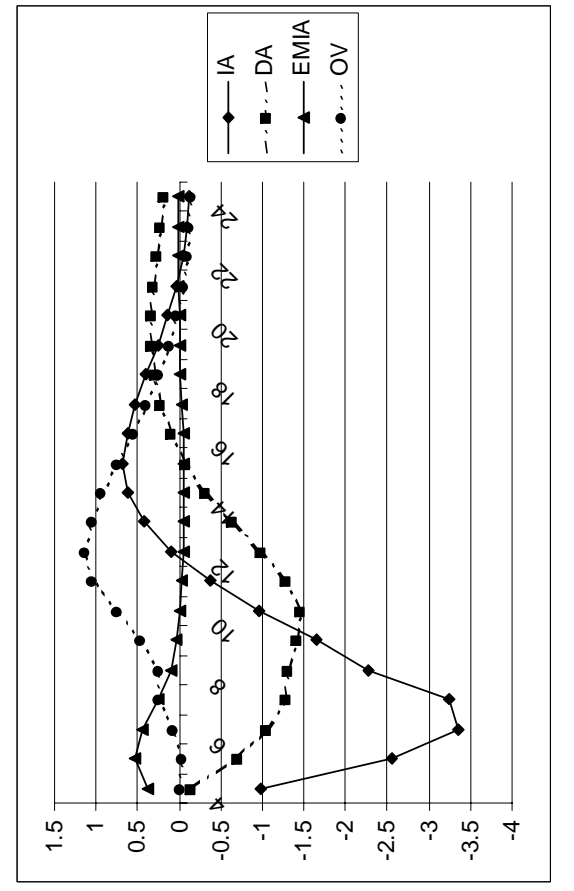

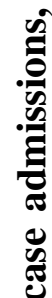

宅

宁

를.

胥

.

.

巳ั:

.

o

을

를

อ․․

๓े

告哇 